\title{
Probing jet medium interactions via $Z+$ jet momentum imbalances
}

\section{Lin Chen, ${ }^{a, *}$ Shu-Yi Wei ${ }^{b, c}$ and Han-Zhong Zhang ${ }^{a}$}

${ }^{a}$ Key Laboratory of Quark and Lepton Physics (MOE) and Institute of Particle Physics, Central China Normal University, Wuhan 430079, China

${ }^{b}$ CPHT, CNRS, École Polytechnique, Institut Polytechnique de Paris, Route de Scalay, 91128 Palaiseau, France

${ }^{c}$ European Centre for Theoretical Studies in Nuclear Physics and Related Areas(ECT*) and Fondazione Bruno Kessler, Strada delle Tabarelle 286, I-38123 Villazzano (TN), Italy

E-mail: chen.1.raymond@mails.ccnu.edu.cn

The recently developed Resummation improved pQCD approach that provides quantitative extraction of the QGP's transport coefficient using dijet momentum imbalances[1-3], was extended to the $Z+$ jet (and $H+$ jet) process with higher order corrections[4]. Taking into account soft radiations that results in divergent behaviour due to Sudakov type logarithms near the back-toback region, the $p p$ baseline was established by comparing with the $5.02 \mathrm{TeV} Z+$ jet data[5] . Then by implementing the BDMPS-Z[6-10] energy-loss formalism with the OSU hydrodynamic simulation $[11,12]$ in our calculation and a comparison with the $A A$ data, the transport coefficient $\hat{q}$ was extracted. The proceeding provides a summary to the above study.

HardProbes 2020

1-6 June 2020

Austin, Texas

${ }^{*}$ Speaker 


\section{Introduction}

It is well-known that the jet transport coefficient $\hat{q}[13-16]$ which includes the effect of transverse momentum broadening and jet energy-loss, is use to probe the transport properties of the QuarkGluon Plasma created in relativistic heavy-ion collisions at RHIC and the LHC $[17,18]$. Most notable effort by the JET collaboration[19] that uses single inclusive hadron yield suppression along with different energy-loss models have achieved great success in the quantitative extraction of $\hat{q}$. Recent developments on dijet, dihadron(hadron-jet), and photon-jet[1-3, 20, 21] differential observables which utilizes the Sudakov resummation formalism, have provided a whole new perspective in the study of the so-called Jet Quenching phenomena.

In this study, we extend the above formalism to $Z+$ jet correlation, with the advantage of being able to calibrate the amount of lost energy with the neutral $Z$ trigger as compare to dijet observables, and cleaner production mechanisms as oppose to photon triggers which were contaminated by background production. Using the previously developed resummation improved pQCD approach, we established a $p p$ baseline in comparison with the 5.02 TeV CMS Z +jet data[5]. Due to the fact that the medium induced transverse momentum broadening effect gets dwarfed by the overwhelming vacuum Sudakov effect at the LHC energy scale, we focus on using the momentum imbalance distribution that reflects parton energy-loss in our calculation.

We briefly summarise our work on setting the $Z+$ jet baseline with the resummation improved pQCD approach, and extracting the transport coefficient $\hat{q}$ with the BDMPS-Z[6-10] formalism in this proceeding[4].

\section{Vacuum and resummation}

The $Z+$ jet momentum imbalance is defined as the ratio of momentum of the outgoing $Z$ boson and leading-jet $x_{J Z}=P_{J \perp} / P_{Z \perp}$. This simple observable, together with the transverse momentum conservation, results in divergent behaviour near the back-to-back region in conventional perturbative calculation due to the appearance of large Sudakov type logarithms. This region, corresponding to $\Delta \phi \sim \pi$ in the azimuthal distribution, requires the use of the sophisticated $q_{T^{-}}$ resummation technique[22], where $\vec{q}_{T} \equiv \vec{P}_{J \perp}+\vec{P}_{Z \perp}$, that takes into account soft vacuum radiations.

Although resummation can nicely describe data near $\pi$, its cross-section diminishes rapidly at large $q_{T}$, and perturbative expansion becomes important. Thus, in order to give a good description of the momentum imbalance distribution, especially near $x_{J Z} \approx 1$, both resummation and perturbative calculation have to be taken into account.

$$
\frac{1}{\sigma} \frac{d \sigma_{\text {improved }}}{d x_{J}}=\left.\frac{1}{\sigma_{\mathrm{pQCD}}} \frac{d \sigma_{\mathrm{pQCD}}}{d x_{J}}\right|_{\Delta \phi<\phi_{m}}+\left.\frac{1}{\sigma_{\mathrm{res}}} \frac{d \sigma_{\mathrm{res}}}{d x_{J}}\right|_{\phi_{m}<\Delta \phi<\pi}
$$

By employing the elegantly effective resummation improved pQCD approach, we slice the azimuthal phase space with a $\phi_{m}$ parameter, and calculate the momentum imbalance distribution using the formalism that suits best in their respective regions. In theory, both formalism should coincide when the order of calculation is high enough, thus the choice of $\phi_{m}$ is insensitive to the numerical results. 

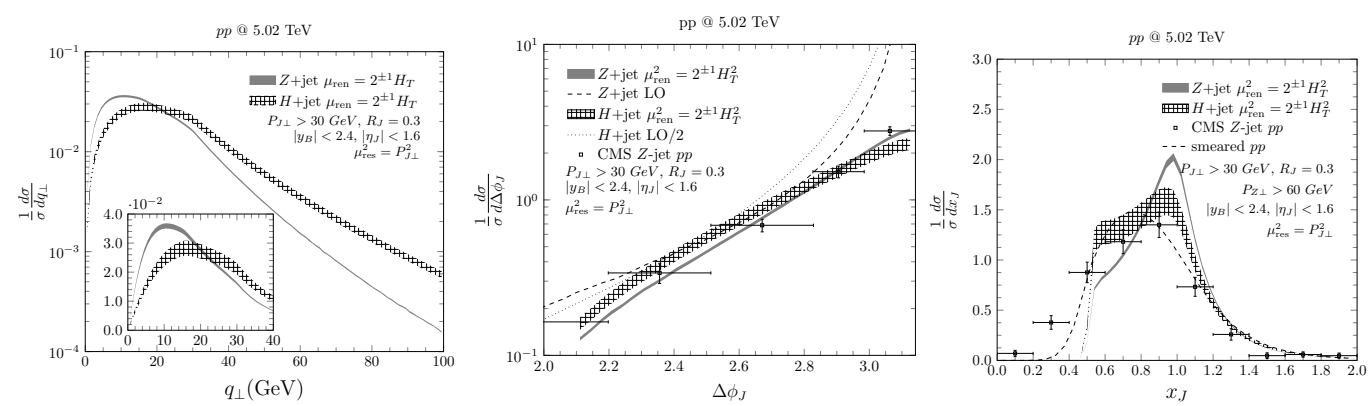

Figure 1: Normalized $q_{\perp}$ (left), $\Delta \phi$ (middle) and $x_{J}$ (right) distribution for both $Z+$ jet(solid) and $H+$ jet(dashdotted) processes at $5.02 \mathrm{TeV}$ in comparison with the CMS experimental data[5]. The resummation scale is fixed at $\mu_{\text {res }}^{2}=P_{J \perp}^{2}$, while the renormalization scale varies by a factor $\mu_{\text {ren }}^{2}=2^{ \pm 1} H_{T}^{2}$.

In our study, both $Z+$ jet and Higgs $(H)+$ jet hard kernels were used in comparison to study the effects and strength of the Sudakov factor. Because observables associated with jet energy is sensitive to detector response effects, which could dramatically change the shape of the $x_{J}$ distribution, and since the unfolded data were not available at the time, we implemented a simple Gaussian smearing function in our calculation to mimic the behaviour of detector effect.

$$
\frac{d \sigma_{\text {smeared }}}{d P_{J \perp}}=\left.\int \frac{d r}{\sqrt{2 \pi} \sigma} e^{-\frac{(r-\bar{r})^{2}}{2 \sigma^{2}}} \frac{1}{r} \frac{d \sigma}{d P_{J \perp}^{\prime}}\right|_{P_{J \perp}=r P_{J \perp}^{\prime}}
$$

where $\bar{r}$ and $\sigma$ depends on the detector and were determined to best fit the current experimental data. Our calculations (Fig. 1) showed that with the kinematic cuts used in the CMS $x_{J Z}$ distribution i.e. $\Delta \phi>7 \pi / 8$, the momentum imbalance is dominated by processes in the back-toback configuration that utilizes the $q_{T}$-resummation formalism.

\section{Medium and energy-loss}

Base on the above $p p$ baseline, we then simulate the space-time evolution of the QGP medium using the OSU 2+1D viscous hydrodynamic code[11, 12], along with a simple temperature scaling parametrization of the transport coefficient to generate a space-time medium induced broadening profile. Integrating over the transverse plane by employing the BDMPS-Z[6-9] energy-loss formalism, we obtained the quenched distribution.

$$
\frac{d \sigma_{A A}}{d P_{J \perp}}=\left.\int d x d y d \psi \frac{T_{A B}(x, y)}{2 \pi} \int d \epsilon D\left(\epsilon, \omega_{c}\left(x, y, \psi ; \hat{q}_{0}\right)\right) \frac{d \sigma_{p p}}{d P_{J \perp}^{\prime}}\right|_{P_{J \perp}=P_{J \perp}^{\prime}-\epsilon}
$$

where $T_{A B}$ is the overlapping normalization factor, and $D$ the radiation probability with a given lost energy $\epsilon$ is defined by BDMPS-Z: $\epsilon D(\epsilon)=\sqrt{\frac{\alpha^{2} \omega_{c}}{2 \epsilon}} \exp \left[-\frac{\pi \alpha^{2} \omega_{c}}{2 \epsilon}\right] . \omega_{c}$ the radiation frequency is the propagation path integral of the transport coefficient $\hat{q}$. Our study (Fig. 2) shows that the values of $\hat{q}_{0}=4 \sim 8 \mathrm{GeV}^{2} / \mathrm{fm}$ gives a good description to the CMS 5.02 TeV AA data.

The extracted values of $\hat{q}$ is in consistency with the values extracted in our previous photon+jet study[3] at $5.02 \mathrm{TeV}$, our dijet study at 2.76 TeV[2] and the original BDMPS estimate at 250 MeV[7]. 


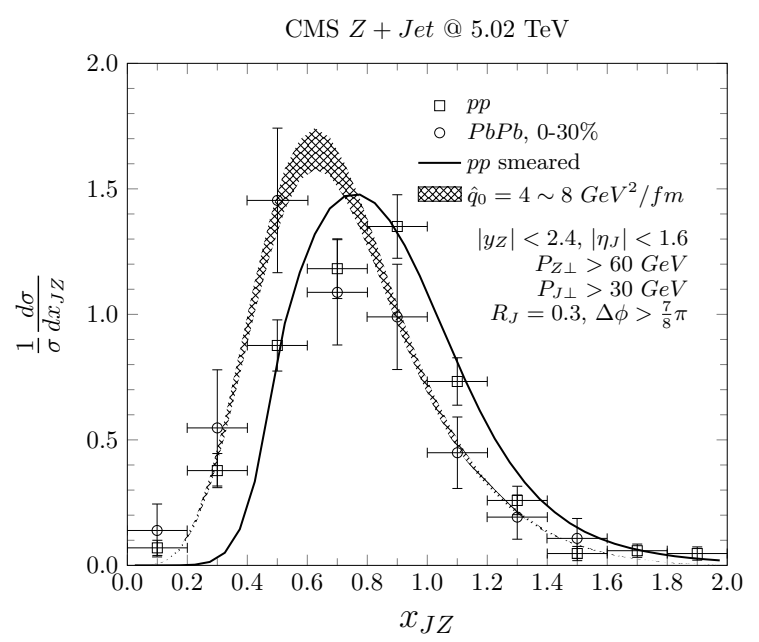

Figure 2: Normalized smeared $x_{J Z}$ distribution for both $p p$ (dashed) and central $P b P b 0-30 \%$ (dotted) data at $5.02 \mathrm{TeV}$ in comparison with the CMS[5] experimental data.

We have also provided calculations for the $Z+$ jet and $H+$ jet momentum imbalance using different $P_{\perp}$ cuts without convoluting with the smearing function (not shown) for future direct comparison with the fully corrected unfolded data.

\section{Summary}

Under the resummation improved pQCD approach, the $p p$ baseline for $Z+$ jet and $H+$ jet were established. The $Z+$ jet calculation were further smeared in comparison with the CMS $p p$ data at $5.02 \mathrm{TeV}$. Then values of $\hat{q}$ were used in conjunction with the BDMPS-Z energy-loss formalism simulated by the OSU hydro, and found that the best fit for the CMS AA data at $5.02 \mathrm{TeV}$ renders $\hat{q}_{0}=4 \sim 8 \mathrm{GeV}^{2} / \mathrm{fm}$. This is in agreement with our previous studies and predictions to the future unfolded $x_{J Z}$ distributions were made. Our study provides a new point of view to the parton energy-loss mechanism in the QGP medium and complements existing Monte-Carlo approaches to momentum imbalance calculations.

\section{Acknowledgements}

This work is supported by Natural Science Foundation of China (NSFC) under grant Nos. 11435004 and 11935007 . S.-Y. W. is supported by the Agence Nationale de la Recherche under the project ANR-16-CE31-0019-02.

\section{References}

[1] L. Chen, G. Y. Qin, S. Y. Wei, B. W. Xiao and H. Z. Zhang, Phys. Lett. B 773, 672 (2017) doi:10.1016/j.physletb.2017.09.031 [arXiv:1607.01932 [hep-ph]].

[2] L. Chen, G. Y. Qin, S. Y. Wei, B. W. Xiao and H. Z. Zhang, Phys. Lett. B 782, 773 (2018) doi:10.1016/j.physletb.2018.06.002 [arXiv:1612.04202 [hep-ph]]. 
[3] L. Chen, G. Y. Qin, L. Wang, S. Y. Wei, B. W. Xiao, H. Z. Zhang and Y. Q. Zhang, Nucl. Phys. B 933, 306 (2018) doi:10.1016/j.nuclphysb.2018.06.013 [arXiv:1803.10533 [hep-ph]].

[4] L. Chen, S. Y. Wei and H. Z. Zhang, [arXiv:2001.07606 [hep-ph]].

[5] A. M. Sirunyan et al. [CMS Collaboration], Phys. Rev. Lett. 119, no. 8, 082301 (2017) doi:10.1103/PhysRevLett.119.082301 [arXiv:1702.01060 [nucl-ex]].

[6] R. Baier, Y. L. Dokshitzer, A. H. Mueller, S. Peigne and D. Schiff, Nucl. Phys. B 483, 291 (1997) doi:10.1016/S0550-3213(96)00553-6 [hep-ph/9607355].

[7] R. Baier, Y. L. Dokshitzer, A. H. Mueller, S. Peigne and D. Schiff, Nucl. Phys. B 484, 265 (1997) doi:10.1016/S0550-3213(96)00581-0 [hep-ph/9608322].

[8] R. Baier, Y. L. Dokshitzer, A. H. Mueller and D. Schiff, Nucl. Phys. B 531, 403 (1998) doi:10.1016/S0550-3213(98)00546-X [hep-ph/9804212].

[9] R. Baier, Y. L. Dokshitzer, A. H. Mueller and D. Schiff, JHEP 0109, 033 (2001) doi:10.1088/11266708/2001/09/033 [hep-ph/0106347].

[10] B. G. Zakharov, JETP Lett. 63, 952 (1996) doi:10.1134/1.567126 [hep-ph/9607440].

[11] H. Song and U. W. Heinz, Phys. Rev. C 77, 064901 (2008) doi:10.1103/PhysRevC.77.064901 [arXiv:0712.3715 [nucl-th]].

[12] Z. Qiu, C. Shen and U. Heinz, Phys. Lett. B 707, 151 (2012) doi:10.1016/j.physletb.2011.12.041 [arXiv:1110.3033 [nucl-th]].

[13] A. Majumder and M. Van Leeuwen, Prog. Part. Nucl. Phys. 66, 41 (2011) doi:10.1016/j.ppnp.2010.09.001 [arXiv:1002.2206 [hep-ph]].

[14] G. Y. Qin and X. N. Wang, Int. J. Mod. Phys. E 24, no. 11, 1530014 (2015) doi:10.1142/S0218301315300143, 10.1142/9789814663717_0007 [arXiv:1511.00790 [hep-ph]].

[15] J. P. Blaizot and Y. Mehtar-Tani, Int. J. Mod. Phys. E 24, no. 11, 1530012 (2015) doi:10.1142/S021830131530012X [arXiv:1503.05958 [hep-ph]].

[16] X. N. Wang and M. Gyulassy, Phys. Rev. Lett. 68, 1480 (1992). doi:10.1103/PhysRevLett.68.1480

[17] B. Muller, J. Schukraft and B. Wyslouch, Ann. Rev. Nucl. Part. Sci. 62, 361 (2012) doi:10.1146/annurevnucl-102711-094910 [arXiv:1202.3233 [hep-ex]].

[18] M. Gyulassy and L. McLerran, Nucl. Phys. A 750, 30 (2005) doi:10.1016/j.nuclphysa.2004.10.034 [nucl-th/0405013].

[19] K. M. Burke et al. [JET Collaboration], Phys. Rev. C 90, no. 1, 014909 (2014) doi:10.1103/PhysRevC.90.014909 [arXiv:1312.5003 [nucl-th]].

[20] A. H. Mueller, B. Wu, B. W. Xiao and F. Yuan, Phys. Lett. B 763, 208 (2016) doi:10.1016/j.physletb.2016.10.037 [arXiv:1604.04250 [hep-ph]].

[21] A. H. Mueller, B. Wu, B. W. Xiao and F. Yuan, Phys. Rev. D 95, no. 3, 034007 (2017) doi:10.1103/PhysRevD.95.034007 [arXiv:1608.07339 [hep-ph]].

[22] P. Sun, B. Yan, C.-P. Yuan and F. Yuan, Phys. Rev. D 100, no. 5, 054032 (2019) doi:10.1103/PhysRevD.100.054032 [arXiv:1810.03804 [hep-ph]]. 